Supplement of Earth Syst. Dynam., 8, 817-826, 2017

https://doi.org/10.5194/esd-8-817-2017-supplement

(C) Author(s) 2017. This work is distributed under

the Creative Commons Attribution 3.0 License.

(c) (i)

Earth System
Dynamics

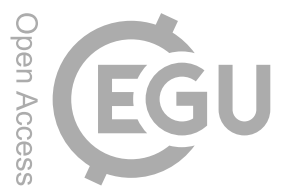

Supplement of

\title{
Effects of the 2014 major Baltic inflow on methane and nitrous oxide dynamics in the water column of the central Baltic Sea
}

\section{Jukka-Pekka Myllykangas et al.}

Correspondence to: Jukka-Pekka Myllykangas (jukka-pekka.myllykangas@helsinki.fi)

The copyright of individual parts of the supplement might differ from the CC BY 3.0 License. 
This document contains the following supplementary materials:

Figure S1 - Establishment of the cut-off values below which data for $\mathrm{CH}_{4}$ and $\mathrm{N}_{2} \mathrm{O}$ are indistinguishable from blanks

Text S2 - Precision and accuracy of the reported data

Figure S2 - Diffusion experiment for gas loss from plastic syringes related to text S2.

Figure S3 - Figure exhibiting the changes in water column salinity at the Western Gotland Basin over a period of 16 years.

Table S4 - Table containing detailed information about the specific sampling sites, depths and coordinates. 


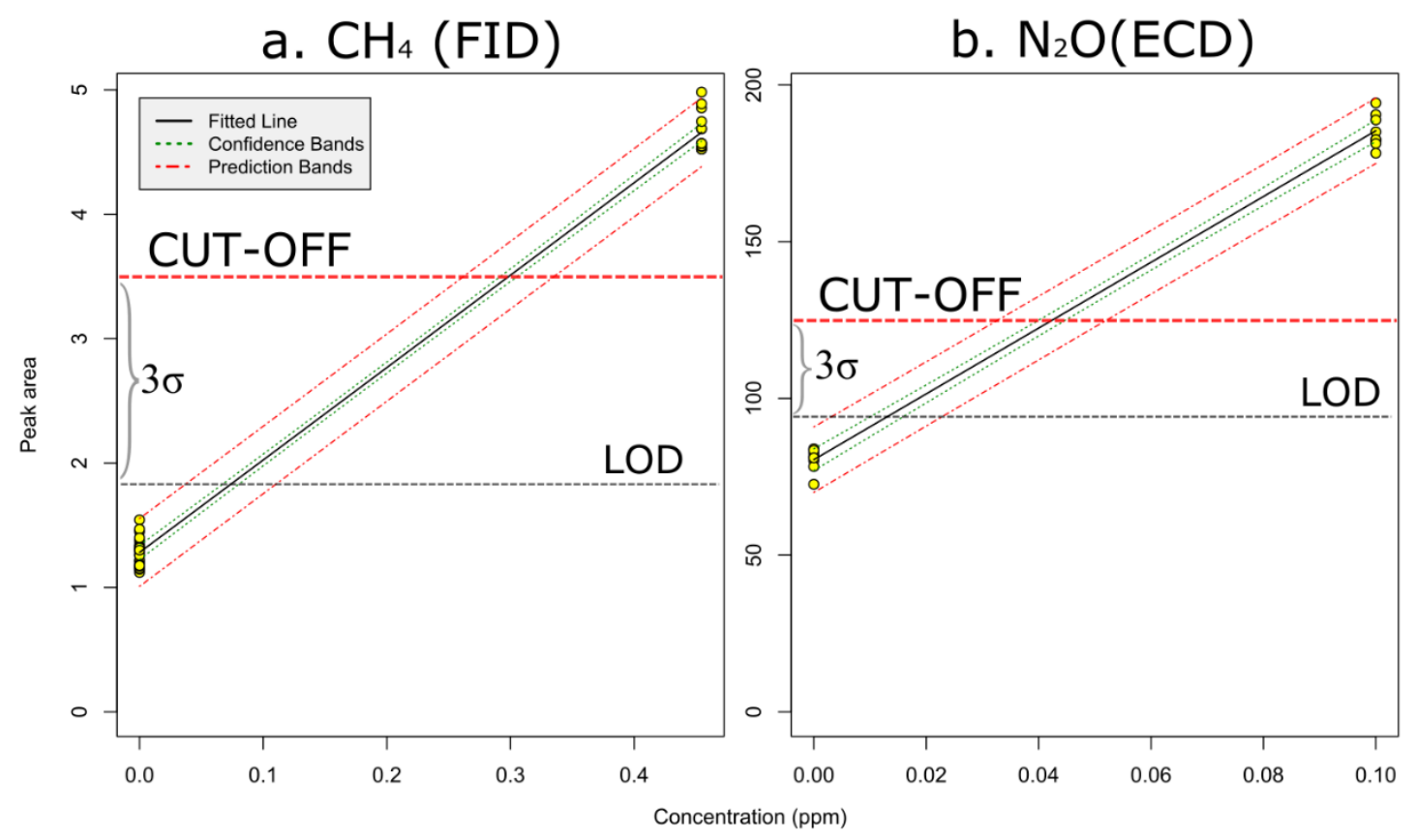

Figure S1. Establishment of cut-off concentrations of $\mathrm{CH}_{4}$ (left) and $\mathrm{N}_{2} \mathrm{O}$ (right), below which measured sample values are regarded as indistinguishable from those of blank pre-evacuated Exetainers. Note the different y-axis scales of the panels, which are due to the different detectors used for the GC measurement of each gas (FID vs. ECD). Yellow points represent measured peak areas of $\mathrm{CH}_{4}$ and $\mathrm{N}_{2} \mathrm{O}$ from blank pre-evacuated Exetainers (i.e. Exetainers from the supplier, subsequently filled with $\mathrm{N}_{2}$, nominal $\mathrm{CH}_{4}$ and $\mathrm{N}_{2} \mathrm{O}$ concentrations $=0 \mathrm{ppm}$ ) and measured peak areas of the lowest standards for each gas, also stored in pre-evacuated Exetainers $\left(\mathrm{CH}_{4}\right.$ and $\mathrm{N}_{2} \mathrm{O}$ concentrations $0.45 \mathrm{ppm}$ and $0.1 \mathrm{ppm}$, respectively). A total of 22 replicate blanks and 14 replicate low standards were measured for $\mathrm{CH}_{4}$ and 8 blanks and 8 low standards for $\mathrm{N}_{2} \mathrm{O}$. The linear fits (peak area vs. ppm) derived from the blanks and the low standards are shown for both gases, with the corresponding 95\% confidence and prediction bands. Note that these fits are linear across the full range of standards (not shown). Limit of detection (LOD) was determined according to Armbruster and Pry $(2008)\left(\right.$ mean $\left._{\text {blank }}+1.645 * \mathrm{SD}_{\text {blank }}\right)+1.645 * \mathrm{SD}_{\text {low standard. To determine the }}$ cut-off, a conservative additional margin was added to the LOD to account for the imprecision of real sample data (in contrast to standard measurements), in which multiple transfers of water and gas between Niskin bottles, syringes and pre-evacuated Exetainers introduce error between replicates. The mean standard deviation in peak area determined from all triplicate samples $(n=N)$ was multiplied by 3 and added to LOD to estimate cut-offs of 3.49 and 125.82 peak area units for $\mathrm{CH}_{4}$ and $\mathrm{N}_{2} \mathrm{O}$, respectively ( $3 \sigma$ in the figure). Mean standard deviation of samples was calculated as the square root of mean variances of all sample triplicates: $\sqrt{\frac{\sum \sigma_{\text {sample }}^{2}}{n_{\text {samples }}}}$. 


\section{Text S2. Precision and accuracy of the reported data}

Here we estimate the precision and accuracy of the reported data (above the cutoff value), caused by the residual air in the Exetainers, the potential effect of diffusive gas exchange in the plastic syringes and other sources of error, including transfer of samples between syringes.

Precision was estimated from triplicate analysis of all samples and was consistently <5\% RSD (see main text). This implies that error introduced by syringe transfers and other sampling artefacts was acceptable for this study where concentrations of $\mathrm{CH}_{4}$ and $\mathrm{N}_{2} \mathrm{O}$ varied over several orders of magnitude.

The average volume of the residual air in the Exetainers was experimentally determined to be $0.7 \pm 0.1 \mathrm{~mL}$ ( $\mathrm{n}=20$, different batches). The manufacturer (LabCo UK) confirmed that the Exetainers are not flushed with any inert gas prior to evacuation, implying that the residual air has atmospheric concentrations of $\mathrm{CH}_{4}$ and $\mathrm{N}_{2} \mathrm{O}$ (mean concentrations $1.834 \mathrm{ppm}, 0.328 \mathrm{ppm}$, respectively (Blasing, 2016)). This introduces a potential source of inaccuracy in the sample data.

When mixing two varying concentrations of a given gaseous substance at constant temperature, the concentration of the resulting mixture can be calculated by mass balance:

$C_{3}=\frac{\left(C_{1} V_{1}\right)+\left(C_{2} V_{2}\right)}{V_{1}+V_{2}}$

The contaminating effect of residual air is expected to be greatest at low sample concentrations, such as those just above the cutoff value (Fig. S1). To estimate the error in accuracy of these samples, let $C_{1}$ be the mean atmospheric $\mathrm{CH}_{4}$ concentration $(1.834 \mathrm{ppm}), V_{l}$ be the mean volume of residual air in the Exetainers $(0.7 \mathrm{~mL}), V_{2}$ the mean volume of the samples $(25 \mathrm{~mL})$ and $C_{3}$ the measured mean concentration of $\mathrm{CH}_{4}$ in the lowest reported samples (approximately $0.4 \mathrm{ppm}$ ). By solving eq. 1 for $C_{2}$, which is the true $\mathrm{CH}_{4}$ concentration in these samples:

$C_{2}=\frac{C_{3}\left(V_{1}+V_{2}\right)-C_{1} V_{1}}{V_{2}}$

it can be calculated that without the contamination, the true mean concentration for a sample measured to contain $0.4 \mathrm{ppm} \mathrm{CH}_{4}$ was in fact $0.35 \mathrm{ppm}$. These calculations suggest that the residual air in the Exetainers causes up to an $11.4 \%$ overestimation for $\mathrm{CH}_{4}$ at the low end of our reported range. For $\mathrm{N}_{2} \mathrm{O}$, the overestimation is $10.1 \%$ for these samples.

When the sample concentration exceed atmospheric concentration, the effect of the residual air switches from enriching to diluting the true concentrations. Using eq 2. for the highest measured samples in this study (30.1 and 13.2 ppm for $\mathrm{CH}_{4}$ and $\mathrm{N}_{2} \mathrm{O}$, respectively) there is a $2.6 \%$ underestimation in $\mathrm{CH}_{4}$ and $2.7 \%$ for $\mathrm{N}_{2} \mathrm{O}$ due to dilution caused by the residual air. The mean concentration in all samples was $2.67 \pm 4.11 \mathrm{ppm}$ for $\mathrm{CH}_{4}$ and $0.24 \pm 0.79 \mathrm{ppm}$ for $\mathrm{N}_{2} \mathrm{O}$, meaning that on average, there is a $0.9 \%$ underestimation in the $\mathrm{CH}_{4}$ samples and a $1.1 \%$ overestimation in the $\mathrm{N}_{2} \mathrm{O}$ samples. We regard these values as acceptable for this study where concentrations of $\mathrm{CH}_{4}$ and $\mathrm{N}_{2} \mathrm{O}$ varied over several orders of magnitude.

The plastic syringes used in sampling (BD Plastipak, $60 \mathrm{~mL}$ Luer-Lok) are made of non-gas tight polypropylene, meaning that diffusive exchange with outside air is a potential further source of inaccuracy in the data. To test this, we conducted an experiment in which 15 syringes were filled simultaneously with the same standard gas mixture (5 ppm $\mathrm{CH}_{4}, 1.1 \mathrm{ppm} \mathrm{N}_{2} \mathrm{O}$ ) and subsequently measured after transfer to Exetainers in triplicate, at 30 minute intervals for 150 minutes (Fig. S2). Based on this experiment, we can conclude that the gas loss per hour is $<0.5 \%$ for $\mathrm{CH}_{4}$ and approximately $1.6 \%$ for $\mathrm{N}_{2} \mathrm{O}$. 


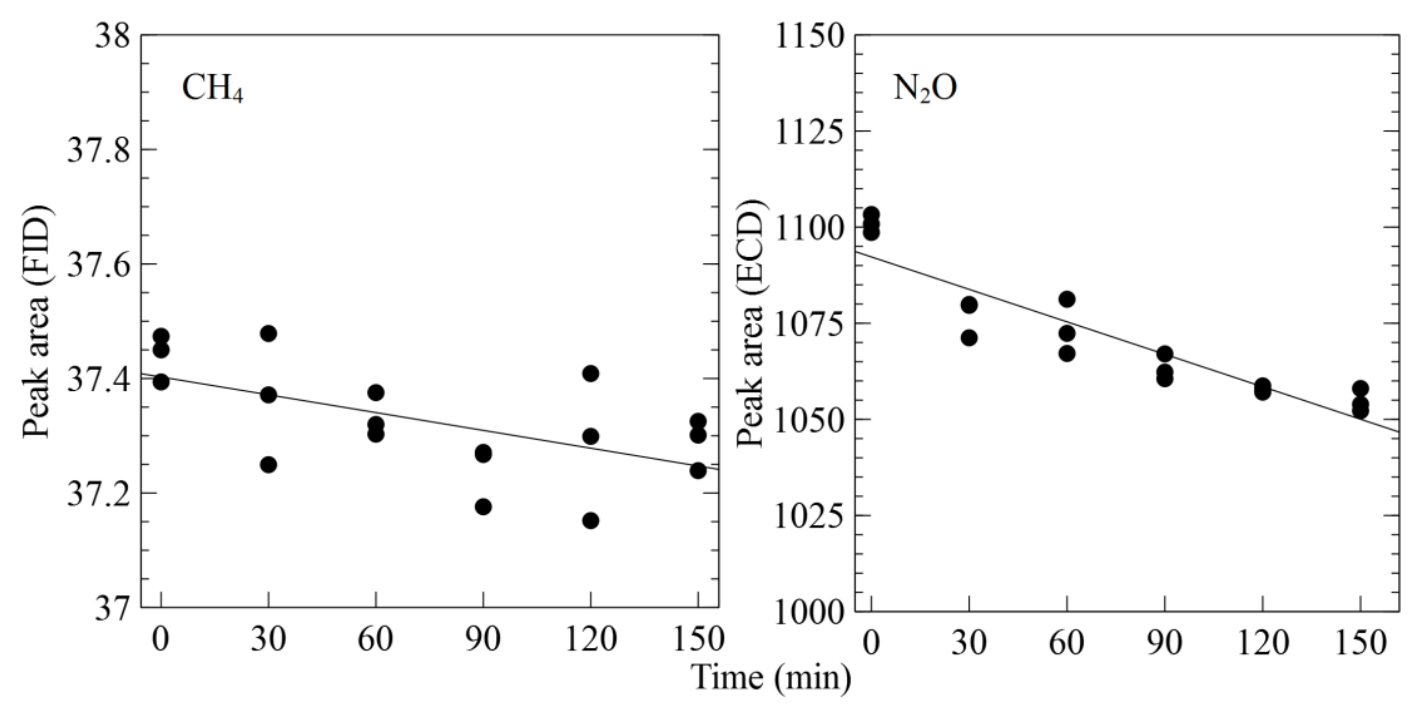

Figure S2. The loss of $\mathrm{CH}_{4}$ and $\mathrm{N}_{2} \mathrm{O}$ from a plastic syringe over 150 minutes in a standard gas mixture containing $5 \mathrm{ppm} \mathrm{CH}_{4}$ and 1 ppm $\mathrm{N}_{2} \mathrm{O}$. Note the different y-axes.

Thus, given that sample gas remained in the syringes for a maximum of 40 minutes, it can be concluded that diffusive exchange was a relatively small factor in the overall inaccuracy $(<1 \%)$.

\section{References:}

Armbruster, D. A. and Pry, T.: Limit of blank, limit of detection and limit of quantitation., Clin. Biochem. Rev., 29 Suppl 1(August), S49-52 [online] Available from:

http://www.pubmedcentral.nih.gov/articlerender.fcgi?artid=2556583\&tool=pmcentrez\&rendertype $=$ abstract, 2008 .

Blasing, T. J.: Recent Greenhouse Gas Concentrations (Updated April 2016), Carbon Dioxide Inf. Anal. Cent., doi:10.3334/CDIAC/atg.032, 2016. 


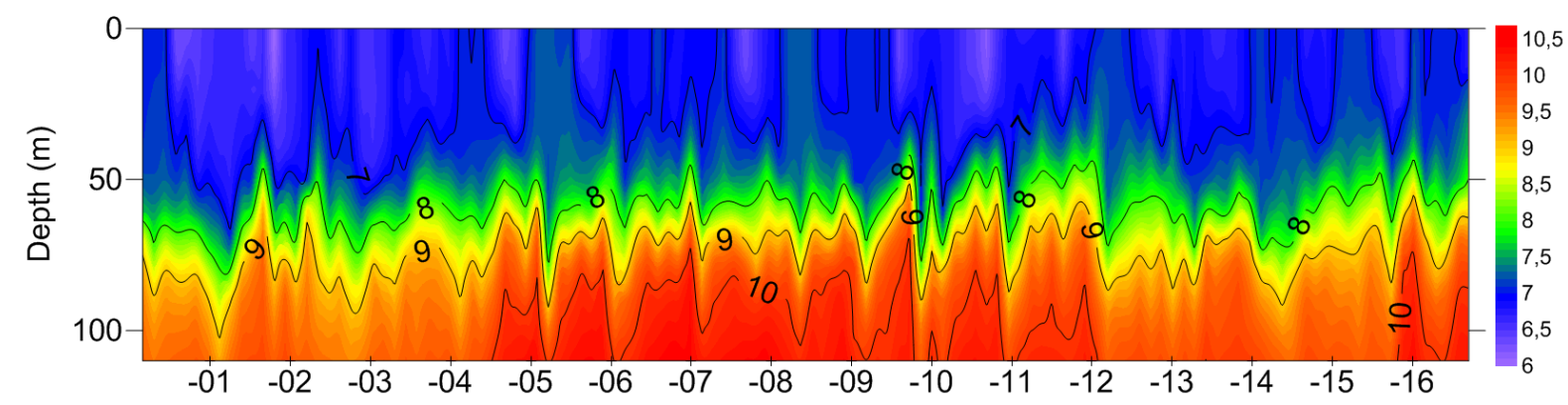

Figure S3. Water column salinity at Western Gotland Basin station BY38 from 2000 to 2016, based on CTD salinity data retrieved from the SMHI Shark database

(http://www.smhi.se/klimatdata/oceanografi/havsmiljodata/marina-miljoovervakningsdata), exhibiting the typical annual to multi-annual oscillations in halocline depth in this area. These oscillations were likely contributing to the changes in the halocline depth observed during 2015 in the Western Gotland Basin. 
Table S4. Sampling dates and depths of gas samples, along with exact sampling station coordinates in decimal minutes (WGS 84).

\begin{tabular}{|c|c|c|c|c|c|c|c|c|c|c|c|c|c|c|c|c|c|c|c|c|c|c|c|c|c|c|c|c|c|c|c|c|c|c|c|c|c|c|}
\hline Sampling & & Coordinat & es (D M.m) & & Max depth & Samp & apling & g dep & ths & & & & & & & & & & & & & & & & & & & & & & & & & & & & & \\
\hline Date & Site & Long. & Lat. & Ship / org. & $(\mathrm{m})$ & 20 & 40 & & 60 & & 80 & 901 & 100 & $105 \quad 109$ & 110 & 115 & 120 & 125 & 130 & 135 & 140 & $142 \quad 143$ & 3144 & 145 & 150 & 155 & 160 & 165167 & 7170 & 175 & 185 & 190 & 192195 & 196 & 200 & & 2362 & 237238 \\
\hline 17.3 .2015 & BY20 & 5759.89 & $\mathrm{~N} 01952.73 \mathrm{E}$ & Aranda / SMHI & 195 & $\mathrm{x}$ & $\mathrm{x}$ & & $\mathrm{x}$ & $\mathrm{x}$ & $\mathrm{x}$ & $\mathrm{x}$ & $\mathrm{x}$ & & & & & $\mathrm{x}$ & & & & & & & $\mathrm{x}$ & & & & & $\mathrm{x}$ & & $\mathrm{x}$ & & & & & & \\
\hline 17.3 .2015 & BY15 & 5718.73 & $\mathrm{~N} 02004.57 \mathrm{E}$ & Aranda / SMHI & 239 & $\mathrm{x}$ & & & $\mathrm{x}$ & $\mathrm{x}$ & $\mathrm{x}$ & $\mathrm{x}$ & $\mathrm{x}$ & & & & & $\mathrm{x}$ & & & & & & & $\mathrm{x}$ & & & & & $\mathrm{x}$ & $\mathrm{x}$ & $\mathrm{x}$ & $\mathrm{x}$ & & $\mathrm{x}$ & $\mathrm{x}$ & & $\mathrm{x}$ \\
\hline 17.3.2015 & BY10 & 5638.02 & N $01935.09 \mathrm{E}$ & Aranda / SMHI & 145 & $\mathrm{x}$ & $\mathrm{x}$ & & $\mathrm{x}$ & $\mathrm{x}$ & $\mathrm{x}$ & $\mathrm{x}$ & $\mathrm{x}$ & & & & & $\mathrm{x}$ & & & & & & & & & & & & & & & & & & & & \\
\hline 22.3.2015 & BY32 & 5801.01 & $\mathrm{~N} 01759.07 \mathrm{E}$ & Aranda / SMHI & 202 & $\mathrm{x}$ & $\mathrm{x}$ & $\mathrm{x}$ & $\mathrm{x}$ & $\mathrm{x}$ & $\mathrm{x}$ & $\mathrm{x}$ & $\mathrm{x}$ & & & & & $\mathrm{x}$ & & & & & & & $\mathrm{x}$ & & & & & $\mathrm{x}$ & & & & & & & & \\
\hline 22.3 .2015 & BY38 & 5707.03 & $\mathrm{~N} 01740.12 \mathrm{E}$ & Aranda / SMHI & 110 & $\mathrm{x}$ & $\mathrm{x}$ & $\mathrm{x}$ & $\mathrm{x}$ & $\mathrm{x}$ & $\mathrm{x}$ & $\mathrm{x}$ & $\mathrm{x}$ & $\mathrm{x}$ & & & & & & & & & & & & & & & & & & & & & & & & \\
\hline 21.4.2015 & BY20 & 5759.89 & N $01952.74 \mathrm{E}$ & Aranda / SMHI & 195 & $\mathrm{x}$ & $\mathrm{x}$ & & $\mathrm{x}$ & $\mathrm{x}$ & $\mathrm{x}$ & $\mathrm{x}$ & $\mathrm{x}$ & & & & & $\mathrm{x}$ & & & & & & & $\mathrm{x}$ & & & & & $\mathrm{x}$ & & $\mathrm{x}$ & & & & & & \\
\hline 21.4 .2015 & BY15 & 5718.89 & $\mathrm{~N} 01956.03 \mathrm{E}$ & Aranda/SMHI & 225 & $\mathrm{x}$ & & & $\mathrm{x}$ & $\mathrm{x}$ & $\mathrm{x}$ & $\mathrm{x}$ & $\mathrm{x}$ & & & & & $\mathrm{x}$ & $\mathrm{x}$ & $\mathrm{x}$ & $\mathrm{x}$ & & & $\mathrm{x}$ & $\mathrm{x}$ & & & & & $\mathrm{x}$ & & & & & $\mathrm{x}$ & $\mathrm{x}$ & & \\
\hline 21.4.2015 & BY10 & 5638.02 & N $01935.09 \mathrm{E}$ & Aranda / SMHI & 144 & $\mathrm{x}$ & $\mathrm{x}$ & & $\mathrm{x}$ & $\mathrm{x}$ & $\mathrm{x}$ & $\mathrm{x}$ & $\mathrm{x}$ & & & & & $\mathrm{x}$ & & & & $\mathrm{x}$ & & & & & & & & & & & & & & & & \\
\hline 26.4.2015 & BY32 & 5801.01 & $\mathrm{~N} 01759.07 \mathrm{E}$ & Aranda / SMHI & 201 & $\mathrm{x}$ & $\mathrm{x}$ & $\mathrm{x}$ & $\mathrm{x}$ & $\mathrm{x}$ & $\mathrm{x}$ & $\mathrm{x}$ & $\mathrm{x}$ & & & & & $\mathrm{x}$ & & & & & & & $\mathrm{x}$ & & & & & $\mathrm{x}$ & & & & $\mathrm{x}$ & & & & \\
\hline 26.4 .2015 & BY38 & 5707.03 & $\mathrm{~N} 01740.12 \mathrm{E}$ & Aranda / SMHI & 110 & $\mathrm{x}$ & $\mathrm{x}$ & $\mathrm{x}$ & $\mathrm{x}$ & $\mathrm{x}$ & $\mathrm{x}$ & $\mathrm{x}$ & $\mathrm{x}$ & $\mathrm{x}$ & & & & & & & & & & & & & & & & & & & & & & & & \\
\hline 4.6 .2015 & BY32 & 5759.99 & N $01759.81 \mathrm{E}$ & Aranda / SYKE & 171 & $\mathrm{x}$ & $\mathrm{x}$ & $\mathrm{x}$ & $\mathrm{x}$ & $\mathrm{x}$ & $\mathrm{x}$ & $\mathrm{x}$ & $\mathrm{x}$ & & & & & $\mathrm{x}$ & & & & & & & $\mathrm{x}$ & & & & $\mathrm{x}$ & & & & & & & & & \\
\hline 5.6.2015 & BY20 & 5800.00 & N $01953.81 \mathrm{E}$ & Aranda / SYKE & 193 & $\mathrm{x}$ & $\mathrm{x}$ & & $\mathrm{x}$ & $\mathrm{x}$ & $\mathrm{x}$ & $\mathrm{x}$ & $\mathrm{x}$ & & & & & $\mathrm{x}$ & & & & & & & $\mathrm{x}$ & & & & & $\mathrm{x}$ & & & & $\mathrm{x}$ & & & & \\
\hline 5.6.2015 & BY15 & 5719.20 & $\mathrm{~N} 02003.00 \mathrm{E}$ & Aranda / SYKE & 239 & $\mathrm{x}$ & & & $\mathrm{x}$ & $\mathrm{x}$ & $\mathrm{x}$ & $\mathrm{x}$ & $\mathrm{x}$ & $\mathrm{x}$ & $\mathrm{x}$ & $\mathrm{x}$ & $\mathrm{x}$ & $\mathrm{x}$ & $\mathrm{x}$ & $\mathrm{x}$ & $\mathrm{x}$ & & & & $\mathrm{x}$ & & & & & $\mathrm{x}$ & & & & & $\mathrm{x}$ & $\mathrm{x}$ & & $\mathrm{x}$ \\
\hline 6.6.2015 & BY10 & 5638.00 & $\mathrm{~N} 01935.00 \mathrm{E}$ & Aranda / SYKE & 143 & $\mathrm{x}$ & $\mathrm{x}$ & & $\mathrm{x}$ & $\mathrm{x}$ & $\mathrm{x}$ & $\mathrm{x}$ & $\mathrm{x}$ & & & & & $\mathrm{x}$ & & & & $\mathrm{x}$ & & & & & & & & & & & & & & & & \\
\hline 5.8 .2015 & BY20 & 5800.00 & $\mathrm{~N} 01953.81 \mathrm{E}$ & Aranda / SYKE & 195 & $\mathrm{x}$ & $\mathrm{x}$ & & $\mathrm{x}$ & $\mathrm{x}$ & $\mathrm{x}$ & $\mathrm{x}$ & $\mathrm{x}$ & & & & & $\mathrm{x}$ & & & & & & & $\mathrm{x}$ & & & & & $\mathrm{x}$ & & & $\mathrm{x}$ & & & & & \\
\hline 5.8.2015 & BY15 & 5719.20 & $\mathrm{~N} 02003.00 \mathrm{E}$ & Aranda / SYKE & 238 & $\mathrm{x}$ & & & $\mathrm{x}$ & $\mathrm{x}$ & $\mathrm{x}$ & $\mathrm{x}$ & $\mathrm{x}$ & $\mathrm{x}$ & $\mathrm{x}$ & $\mathrm{x}$ & $\mathrm{x}$ & $\mathrm{x}$ & $\mathrm{x}$ & $\mathrm{x}$ & & & & & $\mathrm{x}$ & & & & & $\mathrm{x}$ & & & & & $\mathrm{x}$ & $\mathrm{x}$ & & $\mathrm{x}$ \\
\hline 6.8 .2015 & BY10 & 5638.00 & $\mathrm{~N} 01935.00 \mathrm{E}$ & Aranda / SYKE & 142 & $\mathrm{x}$ & $\mathrm{x}$ & & $\mathrm{x}$ & $\mathrm{x}$ & $\mathrm{x}$ & $\mathrm{x}$ & $\mathrm{x}$ & & & & & $\mathrm{x}$ & & & & $\mathrm{x}$ & & & & & & & & & & & & & & & & \\
\hline 6.8.2015 & BY38 & 5707.00 & $\mathrm{~N} 01740.00 \mathrm{E}$ & Aranda / SYKE & 109 & $\mathrm{x}$ & $\mathrm{x}$ & $\mathrm{x}$ & $\mathrm{x}$ & $\mathrm{x}$ & $\mathrm{x}$ & $\mathrm{x}$ & $\mathrm{x}$ & $\mathrm{x}$ & & & & & & & & & & & & & & & & & & & & & & & & \\
\hline 7.8.2015 & BY32 & 5759.99 & $\mathrm{~N} 01759.81 \mathrm{E}$ & Aranda / SYKE & 170 & $\mathrm{x}$ & $\mathrm{x}$ & $\mathrm{x}$ & $\mathrm{x}$ & $\mathrm{x}$ & $\mathrm{x}$ & $\mathrm{x}$ & $\mathrm{x}$ & & & & & $\mathrm{x}$ & & & & & & & $\mathrm{x}$ & & & & $\mathrm{x}$ & & & & & & & & & \\
\hline 24.10 .2015 & BY15 & 5719.22 & $\mathrm{~N} 02003.12 \mathrm{E}$ & Salme / TTU & 231 & $\mathrm{x}$ & & & $\mathrm{x}$ & $\mathrm{x}$ & $\mathrm{x}$ & $\mathrm{x}$ & $\mathrm{x}$ & & $\mathrm{x}$ & & $\mathrm{x}$ & $\mathrm{x}$ & $\mathrm{x}$ & $\mathrm{x}$ & & & & & $\mathrm{x}$ & & & & & $\mathrm{x}$ & & & & & $\mathrm{x}$ & $\mathrm{x}$ & & \\
\hline 25.10 .2015 & BY20 & 5759.99 & N $01957.12 \mathrm{E}$ & Salme / TTU & 169 & & & & $\mathrm{x}$ & $\mathrm{x}$ & $\mathrm{x}$ & $\mathrm{x}$ & $\mathrm{x}$ & & & & & $\mathrm{x}$ & & & & & & & $\mathrm{x}$ & & & $\mathrm{x}$ & & & & & & & & & & \\
\hline 8.12 .2015 & BY20 & 5759.89 & $\mathrm{~N} 01952.73 \mathrm{E}$ & Aranda / SMHI & 196 & $\mathrm{x}$ & $\mathrm{x}$ & & $\mathrm{x}$ & $\mathrm{x}$ & $\mathrm{x}$ & $\mathrm{x}$ & $\mathrm{x}$ & & & & & $\mathrm{x}$ & & & & & & & $\mathrm{x}$ & & & & & $\mathrm{x}$ & & & $\mathrm{x}$ & & & & & \\
\hline 8.12 .2015 & BY15 & 5718.73 & $\mathrm{~N} 02004.57 \mathrm{E}$ & Aranda / SMHI & 239 & $\mathrm{x}$ & $\mathrm{x}$ & $\mathrm{x}$ & $\mathrm{x}$ & $\mathrm{x}$ & $\mathrm{x}$ & $\mathrm{x}$ & $\mathrm{x}$ & & & & & $\mathrm{x}$ & & & & & & & $\mathrm{x}$ & $\mathrm{x}$ & $\mathrm{x}$ & $\mathrm{x}$ & $\mathrm{x}$ & $\mathrm{x}$ & & & & & $\mathrm{x}$ & $\mathrm{x}$ & $\mathrm{x}$ & \\
\hline 9.12 .2015 & BY10 & 5638.02 & $\mathrm{~N} 01935.09 \mathrm{E}$ & Aranda / SMHI & 145 & $\mathrm{x}$ & $\mathrm{x}$ & & $\mathrm{x}$ & $\mathrm{x}$ & $\mathrm{x}$ & $\mathrm{x}$ & $\mathrm{x}$ & & & & & $\mathrm{x}$ & & & & & $\mathrm{x}$ & & & & & & & & & & & & & & & \\
\hline 13.12.2015 & BY38 & 5707.03 & $\mathrm{~N} 01740.12 \mathrm{E}$ & Aranda / SMHI & 111 & $\mathrm{x}$ & $\mathrm{x}$ & $\mathrm{x}$ & $\mathrm{x}$ & $\mathrm{x}$ & $\mathrm{x}$ & $\mathrm{x}$ & $\mathrm{x}$ & & $\mathrm{x}$ & & & & & & & & & & & & & & & & & & & & & & & \\
\hline 13.12.2015 & BY32 & 5801.01 & N $01759.07 \mathrm{E}$ & Aranda / SMHI & 202 & $\mathrm{x}$ & $\mathrm{x}$ & $\mathrm{x}$ & $\mathrm{x}$ & $\mathrm{x}$ & $\mathrm{x}$ & $\mathrm{x}$ & $\mathrm{x}$ & & & & & $\mathrm{x}$ & & & & & & & $\mathrm{x}$ & & & & & $\mathrm{x}$ & & & & & $\mathrm{x}$ & & & \\
\hline
\end{tabular}

